\title{
Theoretical determination of the number of adits and cross-sectional area for the airflow requirements of the New Level Mine Project
}

\author{
El Acuña Codelco Chile, Chile \\ G Soto Codelco Chile, Chile
}

\begin{abstract}
The New Level Mine Project (NLMP) underground expansion represents the future of the El Teniente mines in Rancagua, Chile. With the expansion, the daily production rate is expected to be between $137 \mathrm{ktpd}$ and a maximum of $180 \mathrm{ktpd}$, with production planned to start in the end of 2017 or early 2018. The airflow requirements of the project have been estimated in the range of $4,000 \mathrm{~m}^{3} / \mathrm{s}$ when reaching the end of the ramp up, shortly after the year 2025 for 137 ktpd. In order to deliver such volume of fresh airflow the NLMP is considering the construction of several parallel adits for intake and exhaust that will allow the circulation of the required volume. The adits are considered to be built over the upcoming years, with the first pair of adits being under construction, at a cross-sectional area of $7 \times 7 \mathrm{~m}$. Currently, the NLMP is considering the possibility of using larger cross-sections which have not been built before at El Teniente. This paper presents a theoretical exercise developed to determine the optimum number of adits and cross-sectional area to circulate the required airflow, assuming a symmetrical condition and concentrating only in the optimisation of the intake adits. The theoretical exercise consists of expanding the initial development of a single airway economic cross-sectional area into multiple airways economic cross-sectional area based on developing a trade-off between the capital and operational (energy) costs. As the initial development, the approach found in the literature consists of finding the economical balance point between the capital or development cost and the operational or energy cost. The results are presented and discussed with the recommendation made to the NLMP, which is being considered for the detailed engineering phase.
\end{abstract}

\section{Introduction}

During feasibility engineering of the New Level Mine Project (NLMP), and according to the total volume of airflow required to be circulating into the main ventilation system, a certain number of adits was defined, considering that the largest cross-section could be $7 \times 7 \mathrm{~m}$. Adits are horizontal or inclined access tunnels used for the sole purpose of ventilation, whether it is to bring fresh air into the mine or exhaust the return air to the surface.

At the beginning of the detailed engineering and construction phase, and as a result of the additional geotechnical data gathered, the possibility of having larger cross-sections for the intakes and exhaust airways of the project was considered. In order to justify such change, a study was developed to determine the number of adits and their cross-section and to maximise the benefit of the project in terms of balancing the additional capital costs against the energy cost required for the ventilation system over time.

This paper is divided into four sections. First, some of the background to understand the calculation of the pressure drop and energy required to ventilate a tunnel is given, with an explanation of what is an economical cross-section for a tunnel or drift. Second, an explanation is given to understand how to expand the initial economic cross-sectional area model for multiple parallel airways. Third, the gathered data and the extended model are used to determine the number of adits and their cross-sectional areas considering the possibility of having cross-sections larger than $7 \times 7 \mathrm{~m}$ (Figure 1). The results are discussed in terms of the current situation of the project and the impact of performing this analysis at an earlier stage of the 
project such as during the feasibility engineering stage. Finally, conclusions are presented in terms of the recommendation for the project and the gaps that remain to be closed.

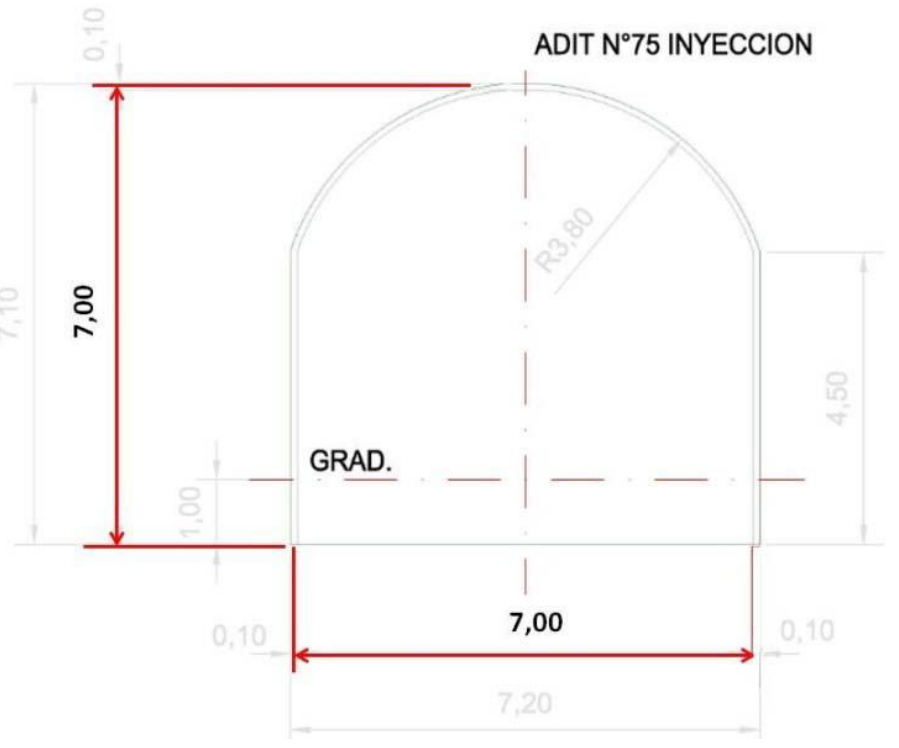

Figure 1 Typical $7 \times 7 \mathrm{~m}$ adit cross-section

\section{Mine ventilation and economic cross-sectional area background}

Mine ventilation is usually divided into two systems: the main or primary ventilation system and the auxiliary or secondary system. The main system is responsible for bringing the fresh air from surface through the intake(s) and distribute it through the network of interconnected underground tunnels, and then back to surface through the exhaust(s). The secondary system takes the fresh air from primary system and then brings it to the work face where most of the contaminants are generated. The main objective of the ventilation system is to dilute and remove the contaminants from the work faces or other places where they are generated, and to maintain a safe and healthy environment underground for the work force.

Once the airflow requirements have been estimated for a certain operation, the number, location and cross-sectional size of intakes and exhausts required are determined. The starting case would be to consider a single intake and a single exhaust; as such the problem is symmetrical. The energy required to move the airflow through the adit is delivered by fan(s) and is a function of the airflow and the pressure drop created by moving the airflow through the adit. Equation (1) presents the Atkinson equation, which indicates how to calculate the pressure drop considering the airflow moving through the adit.

$$
\Delta P=\frac{L \cdot P e r \cdot k}{A^{3}} \cdot Q^{2}
$$

Where:

$$
\begin{aligned}
& \Delta P \quad=\text { the pressure drop }(\mathrm{Pa}) . \\
& A \quad=\text { the cross-sectional area }\left(\mathrm{m}^{2}\right) . \\
& P e r \quad=\text { the perimeter }(\mathrm{m}) . \\
& k \quad=\text { the friction factor of the walls and floor }\left(\mathrm{kg} / \mathrm{m}^{3}\right) . \\
& L \quad=\text { the length of the adit }(\mathrm{m}) . \\
& Q \quad=\text { the airflow circulating through the adit }\left(\mathrm{m}^{3} / \mathrm{s}\right) .
\end{aligned}
$$

Equation (1) should also consider shock losses according to McPherson (1993), but to simplify this exercise, additional losses will not be considered. Accordingly, for this exercise in particular, the impact of shock 
losses was not calculated as it was developed as a first approach, only considering straight adits. The actual geometry considers three $35^{\circ}$ soft bends which are not expected to contribute significantly to the total losses, and hence to the results of this exercise. Equation (2) indicates how to calculate the power dissipated by the airflow circulating through the adit.

$$
P o t=\frac{\Delta P \cdot Q}{\eta}
$$

Where:

$$
\begin{aligned}
& \text { Pot }=\text { the power provided by the fan }(\mathrm{kW}) . \\
& \Delta P=\text { the pressure drop }(\mathrm{kPa}) . \\
& Q \quad=\text { the airflow circulating through the adit }\left(\mathrm{m}^{3} / \mathrm{s}\right) . \\
& \eta \quad=\text { the efficiency of the fan in the adit. }
\end{aligned}
$$

Equation (3) indicates how to calculate the annual energy cost associated with the power required to circulate the airflow through the adit.

$$
C e=\frac{\Delta P \cdot Q}{\eta} \cdot e \cdot H
$$

Where:

$$
\begin{aligned}
& C e \quad=\text { the annual energy cost (USD/year). } \\
& \Delta P \quad=\text { the pressure drop ( } \mathrm{kPa}) . \\
& Q \quad=\text { the airflow circulating through the adit }\left(\mathrm{m}^{3} / \mathrm{s}\right) . \\
& \eta \quad=\text { the efficiency of the fan in the adit. } \\
& e \quad=\text { the energy cost (USD/kWh). } \\
& H \quad=\text { the yearly effective running time of the fan }(\mathrm{s}) \text { in the adit }(\mathrm{h}) .
\end{aligned}
$$

These three equations enable the calculation of the annual cost of the energy required to ventilate the adit, associated only with the ventilation concept of bringing the air from surface to the end of the adit.

As indicated by Acuña and Jodouin (2010), a larger cross-sectional area for a fixed airflow volume generates an exponentially lower energy cost to ventilate the adit. However, this does not come for free, as a larger cross-sectional area has a larger construction cost. As a result, there has to be a point in terms of the cross-sectional area, where a balance is found between the construction or capital cost and the energy or operational cost. This is referred by Hartman et al. (1997) and McPherson (1993) as the economical cross-sectional area. Figure 2 presents a typical graph of an economic cross-sectional analysis.

As can be observed in Figure 2, the balance or minimum total cost is found with the raise or adit diameter

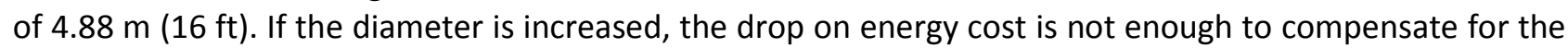
increase in capital required to build the larger raise.

Once raises or adits are built, they are commonly used for several years until the reserves are depleted. As a result the energy consumption is the same year after year. The energy price used in this exercise is fixed for the long-term, fixing the energy cost that has to be discounted as a cash flow to make it comparable with the capital cost usually spent in the first years. In order to achieve that objective, the energy and capital costs are discounted using the net present value (NPV) concept. To make a conservative approximation the capital cost is assumed to be spent over the first year and hence is not discounted. Normally an adit takes between two to three years to be developed, as such the capital cost could be divided in two or three and the second and third year capital cost should be discounted as well as part of the cash flow. Capital cost is calculated based on the average cost per metre multiplied by the length of the 
adits. The average cost per metre is calculated as a weighted average of the cost for each rock quality and the number of metres expected for each rock quality. Equation (4) presents the annuity of the NPV, in which the cash flow or energy cost will be the same each year as indicated by Acuña and Jodouin (2010).

$$
N P V a=\frac{F}{r} \cdot\left(1-\frac{1}{(1+r)^{n}}\right)
$$

Where:

$$
\begin{aligned}
& N P V a=\text { the net present value of a constant cash flow (USD). } \\
& F \quad=\text { the yearly cash flow (in this case energy cost) (USD). } \\
& r \quad=\text { the discount rate. } \\
& n \quad=\text { the number of periods. }
\end{aligned}
$$

\section{Economical analysis}

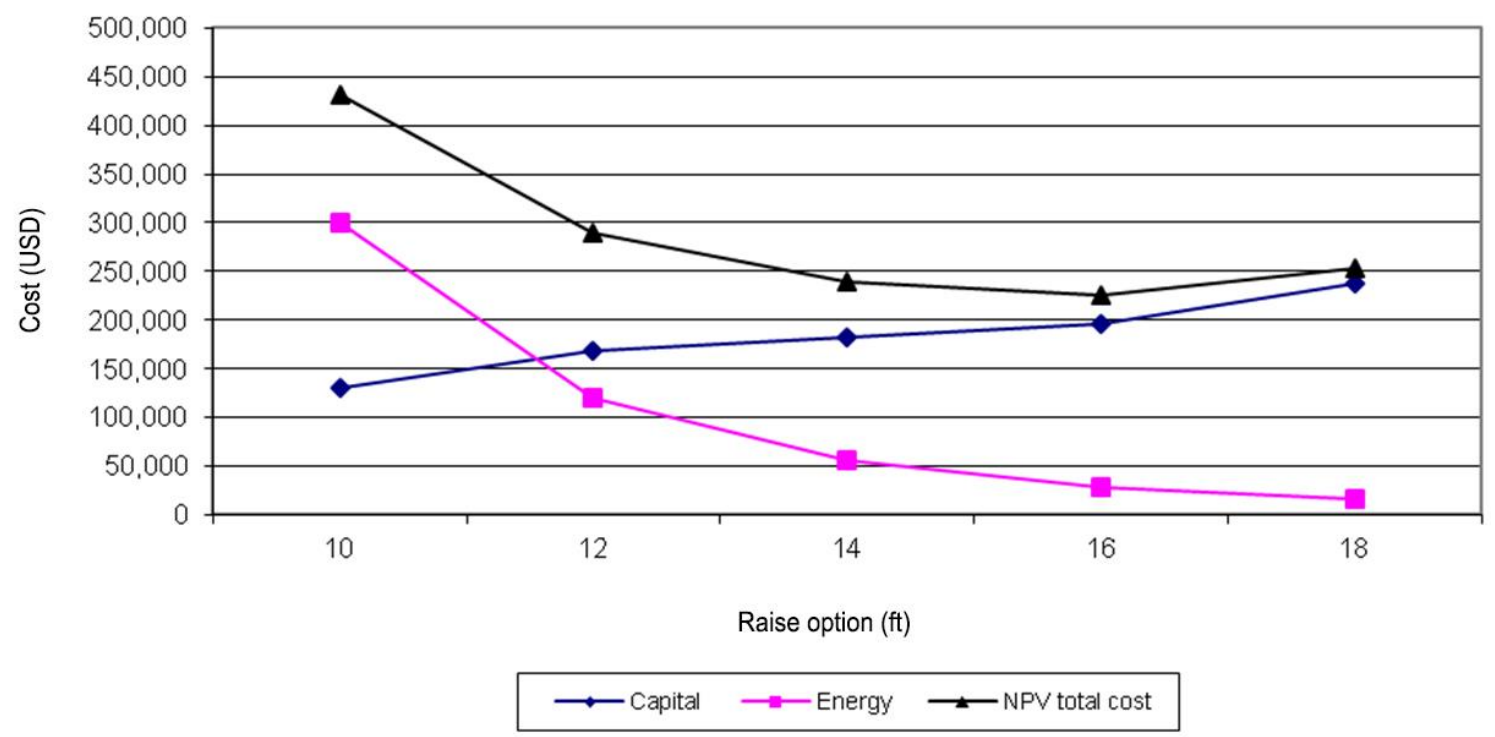

Figure 2 Typical economical analysis graph (Acuña \& Jodouin 2010)

\section{Economic cross-sectional area for multiple parallel airways}

The initial model for economic cross-sectional area presented by McPherson (1993), only considers the optimisation of a single airway. Hartman et al. (1997) also presents a model for multiple airways but constrained to a fix cross-sectional area. However when multiple parallel intakes or exhausts and multiple cross-sectional sizes are considered, the initial model has to be used in a way that allows for selecting the economical cross-section of multiple intakes, exploring different cross-sectional sizes, that will deliver the total required airflow volume.

Certain assumptions have to be made at this point. First, the airflow volume entering the mine and exiting the mine is virtually the same amount, hence defining the number of intakes and exhausts can be approached as a symmetrical problem. Second, the total volume of airflow entering or exiting the mine will have to be evenly divided by the number of parallel airways that will operate as intake or exhaust, assuming that each airway will have the same cross-sectional size and hence the same resistance to the flow resulting on an even split. As a result of the second assumption, each airway will carry the same airflow volume and also the size of the cross-section will have to be optimal in terms of that particular airflow. 
Currently the cross-section defined for the adits of the project is $7 \times 7 \mathrm{~m}$, if those dimensions are input into the cross-sectional analysis, an economic velocity and airflow for that cross-section can be determined, and as such for any other cross-section. Equation (5) enables the calculation of the airflow considering the cross-sectional area and the mean velocity of the airflow.

$$
Q=v \cdot A
$$

Where:

$$
\begin{array}{ll}
Q= & \text { the airflow volume }\left(\mathrm{m}^{3} / \mathrm{s}\right) . \\
v= & \text { the velocity of the airflow }(\mathrm{m} / \mathrm{s}) . \\
A= & \text { the cross-sectional area }\left(\mathrm{m}^{2}\right) .
\end{array}
$$

The economic velocity will be a function of the balance between the capital and the energy cost discounted cash flow. The NLMP is considering a total airflow circulation of $4,000 \mathrm{~m}^{3} / \mathrm{s}$. The total airflow volume of the mine can be divided by the airflow that each economic cross-sectional area can carry. As a result there will be a number of adits, each one with the same cross-sectional area and an economic airflow that they can carry. In the case of the $7 \times 7 \mathrm{~m}$, it was initially determined that the economic airflow was about $500 \mathrm{~m}^{3} / \mathrm{s}$, and eight intake adits were required thus, to circulate a total airflow of $4,000 \mathrm{~m}^{3} / \mathrm{s}$.

However, if different dimensions for the adits are considered, then each cross-section will have an economical airflow volume that they can carry and an associated energy cost. Also depending on the economical airflow per adit, the number of adits will vary in order to deliver the $4,000 \mathrm{~m}^{3} / \mathrm{s}$.

This will generate multiple solutions, one for each cross-section considered, which will allow the generation of an equivalent analysis like the economical cross-section but for multiple airways or adits.

It is expected that as the airflow volume increases through the adits, the energy cost will increase, but there will be a point or airflow volume at which one less adit will be required to provide the total airflow volume. At this point the number of adits required will be one less; hence the construction or capital cost will be reduced. Also, as the airflow volume increases through the adits, the economical cross-section will dictate a change, increasing from one cross-section to the next. This change of cross-section with the number of adits considered will generate an important drop in the energy cost for ventilation, while increasing the construction cost. As a result of these changes, which will generate trade-offs between capital and energy cost, it is expected that for a certain total volume considered, a balance has to be found between capital and energy cost. This is the fundamental objective function that defines the economical cross-section, however, constraints in this model not only limit to only one airway, but to multiple parallel airways. In that regard, the economical cross-section analysis would be a particular case of the analysis that this study attempted to perform.

\section{$4 \quad$ Multiple parallel economic cross-sectional area manual methodology}

The methodology used during this study, was divided into the following steps:

1. Determine the different available cross-sectional areas for the analysis. Basically, take into account the total cross-section considered to be built within the scope of the project (also consider potential constraints for cross-section sizes in certain areas).

2. Determine the cost of developing each cross-sectional area. In this case only an average cost is used for each cross-section area, which is the weighted average of the cost for each rock quality over the total length considered for the adit.

3. Determine the energy cost as a function of the kWh.

4. Determine economical cross-section and energy cost for each airflow volume within the range using the methodology introduced by Hartman et al. (1997) and McPherson (1993). In this case, 
the range is defined by the lower bound of $500 \mathrm{~m}^{3} / \mathrm{s}$, which will be incremented in steps of $10 \mathrm{~m}^{3} / \mathrm{s}$ until the total volume of the airflow is reached for the largest cross-section.

5. Determine the number of adits required for each airflow volume and cross-sectional area in the range of step four to deliver the required total airflow volume and calculate the construction cost of the adits.

6. Determine graphically, the economical airflow, number of adits and cost per each available cross-section.

7. Determine graphically, the recommended economical cross-section and number of adits.

In this exercise, there is no consideration in the capital cost for the purchase of primary fans, as the cost of fans over time is not significant if compared to the energy cost of using them. This multiple parallel economical cross-section analysis was performed for the $3,500 \mathrm{~m}^{3} / \mathrm{s}$ case. In the current case, the NLMP has already started the construction of two (one for intake and one for exhaust) $7 \times 7 \mathrm{~m}$ adits with an estimated length of 2,200 $\mathrm{m}$ to connect the surface with the secondary ventilation system of the mine. These adits will carry $500 \mathrm{~m}^{3} / \mathrm{s}$, and as a result the multiple parallel economical cross-section analysis has to be developed considering the remaining $3,500 \mathrm{~m}^{3} / \mathrm{s}$ to complete the total requirement of $4,000 \mathrm{~m}^{3} / \mathrm{s}$.

The data required for applying the described methodology was obtained from the current developments of the NLMP, which are used to generate Table 1 with the average development (capital) costs for each crosssection and to estimate the energy cost presented in Table 2. Table 1 presents the development cost for each cross-sectional area. The energy cost was estimated at 0.101 (USD/kWh), however this value could potentially increase in time because of the current situation of energy availability. The energy cost is not explicitly considered in the development cost for this study. Values in Table 2 are the ones used by the NLMP to develop the study.

\section{Table 1 Cross-section development cost}

\begin{tabular}{ccc}
\hline Height $(\mathrm{m})$ & Width $(\mathrm{m})$ & Capital cost (USD) \\
\hline 5 & 5 & $14,492,027$ \\
6 & 6 & $19,078,784$ \\
7 & 7 & $24,499,496$ \\
8 & 8 & $30,754,164$ \\
9 & 9 & $37,842,788$ \\
10 & 10 & $45,765,368$ \\
11 & 11 & $54,521,903$ \\
12 & 12 & $64,112,395$ \\
13 & 13 & $74,536,842$ \\
\hline
\end{tabular}


Table 2 Additional parameters

\begin{tabular}{ccc}
\hline Item & Value & Units \\
\hline Energy cost & 0.101 & USD $/ \mathrm{kWh}$ \\
Friction factor & 0.0093 & $\mathrm{~kg} / \mathrm{m}^{3}$ \\
Length & 2,200 & $\mathrm{~m}$ \\
Perimeter & 24.08 & $\mathrm{~m}$ \\
Area & 44.6 & $\mathrm{~m}^{2}$ \\
Hours in a year & 8,760 & $\mathrm{~h}$ \\
Discount rate & $8 \%$ & - \\
Years (LOF) & 50 & Year \\
\hline
\end{tabular}

\section{$5 \quad$ Results}

Once the data was obtained, the methodology indicated in the previous section was applied and a spreadsheet was developed for the $3,500 \mathrm{~m}^{3} / \mathrm{s}$ case, with the calculations for each airflow volume starting at $500 \mathrm{~m}^{3} / \mathrm{s}$. The results of the calculations for the $3,500 \mathrm{~m}^{3} / \mathrm{s}$ total airflow volume case are presented in Figure 3 and the summary of the results are presented in Table 3. Table 4 presents the ranges of airflow volume for each economic cross-sectional area.

Table 3 Summary of $3,500 \mathrm{~m}^{3} / \mathrm{s}$ total airflow volume case study

\begin{tabular}{ccccc}
\hline $\begin{array}{c}\text { Cross-section } \\
(\mathrm{m})\end{array}$ & $\begin{array}{c}\text { Adit airflow } \\
\left(\mathrm{m}^{3} / \mathrm{s}\right)\end{array}$ & $\begin{array}{c}\text { Number of } \\
\text { adits }\end{array}$ & $\begin{array}{c}\text { Total airflow } \\
\left(\mathrm{m}^{3} / \mathrm{s}\right)\end{array}$ & Cost (USD) \\
\hline $7 \times 7$ & 500 & 7 & 3,500 & $259,468,360$ \\
$8 \times 8$ & 590 & 6 & 3,540 & $248,070,282$ \\
$9 \times 9$ & 700 & 5 & 3,500 & $238,290,899$ \\
$10 \times 10$ & 880 & 4 & 3,520 & $229,122,546$ \\
$11 \times 11$ & 1,170 & 3 & 3,510 & $213,978,586$ \\
$12 \times 12$ & 1,380 & 3 & 4,140 & $245,877,181$ \\
\hline
\end{tabular}

Table 4 Economic cross-sectional area airflow volume range

\begin{tabular}{ccc}
\hline $\begin{array}{c}\text { Cross-section } \\
(\mathbf{m})\end{array}$ & $\begin{array}{c}\text { Bottom airflow } \\
\left(\mathbf{m}^{3} / \mathbf{s}\right)\end{array}$ & $\begin{array}{c}\text { Upper airflow } \\
\left(\mathbf{m}^{3} / \mathbf{s}\right)\end{array}$ \\
\hline $7 \times 7$ & 500 & 500 \\
$8 \times 8$ & 510 & 670 \\
$9 \times 9$ & 680 & 870 \\
$10 \times 10$ & 880 & 1,100 \\
$11 \times 11$ & 1,110 & 1,370 \\
$12 \times 12$ & 1,380 & 1,580 \\
\hline
\end{tabular}

As can be observed in Figure 3, the energy cost gradually raise as the airflow in the airway increases, and then drops with the change of cross-sectional area. The minimum energy cost can be observed in Figure 3 
for the bottom of the airflow ranges presented in Table 4: 510, 680, 880, 1,110 and 1,380 $\mathrm{m}^{3} / \mathrm{s}$. The development cost is mainly influenced by the number of adits and their cross-section.

As displayed in Figure 3, almost every increase in cross-section area is coupled with an increase in development cost, and every reduction in the number of adits generates a decrease in the development cost. Table 4 indicates at which airflow volume values there is a change in the cross-section area and Table 5 indicates the number of adits according to the range of airflow volume circulating through each. Numbers in Tables 4 and 5 are different as for the same number of adits different cross-sectional area could be an alternative. The only exception is the change from $9 \times 9 \mathrm{~m}$ cross-section to $10 \times 10 \mathrm{~m}$, because at that particular point the change in cross-section is coupled with a reduction in the number of adits, cancelling each other, and resulting in a very little change of the development cost.

\section{Table 5 Airflow volume range per number of adits}

\begin{tabular}{ccc}
\hline $\begin{array}{c}\text { Number of } \\
\text { adits }\end{array}$ & $\begin{array}{c}\text { Bottom airflow } \\
\left(\mathbf{m}^{3} / \mathbf{s}\right)\end{array}$ & $\begin{array}{c}\text { Upper airflow } \\
\left(\mathbf{m}^{3} / \mathbf{s}\right)\end{array}$ \\
\hline 7 & 500 & 580 \\
6 & 590 & 690 \\
5 & 700 & 870 \\
4 & 880 & 1,160 \\
3 & 1,170 & 1,580 \\
\hline
\end{tabular}

The resulting total cost curve tends to mimic the shape of the energy curve with two exceptions: first, at $590 \mathrm{~m}^{3} / \mathrm{s}$ the reduction in the number of adits from seven to six generates and important drop of the cost, second, in the last cross-section change to $12 \times 12 \mathrm{~m}$ the increase in development cost is compensated by the reduction in energy cost.

\section{Methodology results $3,500 \mathrm{~m}^{3} / \mathrm{s}$ case}

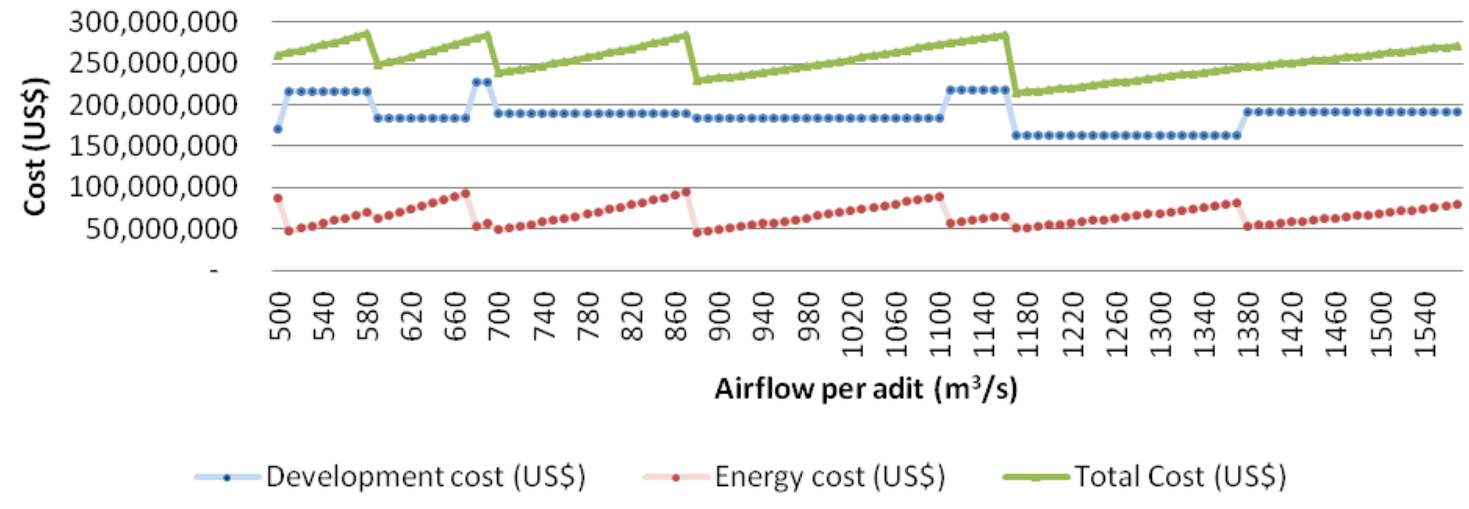

Figure 3 Results of $3,500 \mathrm{~m}^{3} / \mathrm{s}$ total airflow volume case

It is important to note that the strategy of varying the airflow on each airway matches the total airflow requirement only at the points were the number of adits decrease, for all the other cases the airflow is larger than the requirement. As a result the best values for each cross-section are presented in Table 3.

As presented in Table 3 the total cost steadily gets reduced by larger cross-sections, which require less energy, and reduced number of adits which requires less development cost, when meeting the total airflow requirement. Only the $12 \times 12 \mathrm{~m}$ cross-section is more expensive than the previous section because the airflow volume that justifies such dimensions considering three adits overly surpasses the total airflow requirement of $3,500 \mathrm{~m}^{3} / \mathrm{s}$. 
Several observations can be made from the results obtained:

- For the $500 \mathrm{~m}^{3} / \mathrm{s}$ airflow volume, the economical cross-section is the $7 \times 7 \mathrm{~m}$, which validates the choice of the airflow volume for the cross-section resulting from the study developed during the previous engineering phase of the project.

- For the $3,500 \mathrm{~m}^{3} / \mathrm{s}$ total airflow volume, the economical multiple parallel cross-section area is $11 \times 11 \mathrm{~m}$, considering three adits as indicated in Table 3 .

- The minimum airflow required to justify the $12 \times 12 \mathrm{~m}$ cross-section as the economical cross-section, according to the parameters presented in Tables 1 and 2, is $1,380 \mathrm{~m}^{3} / \mathrm{s}$. The minimum numbers of adits required to deliver at least $3,500 \mathrm{~m}^{3} / \mathrm{s}$ is three and the delivered airflow is $4,140 \mathrm{~m}^{3} / \mathrm{s}$, which exceeds by $640 \mathrm{~m}^{3} / \mathrm{s}$ the required airflow and delivers a higher overall cost. As a result the $12 \times 12 \mathrm{~m}$ cross-section is not suggested for airflows in the range of $3,500 \mathrm{~m}^{3} / \mathrm{s}$, and the $11 \times 11 \mathrm{~m}$ cross-section remains the economical cross-section for this multiple parallel economical cross-section manual study.

In the case of the New Level Mine Project, it was defined that within the ground conditions available, the largest cross-section area that could be used for intake and exhaust adits was $9 \times 9 \mathrm{~m}$. As a result the $9 \times 9 \mathrm{~m}$ was selected as the economical cross-section for the upcoming adits remaining to be built.

However, the decision was not straight forward. The result of this study was used as an input for a larger study considering other alternatives such as:

- The construction methodology of using a tunnel boring machine (TBM).

- The additional development cost to place the different number of adits required by each cross-section, within the geographic limitations available on surface.

The TBM is currently considered more costly than the regular drill and blast (D\&B) methodology used at EI Teniente for development according to the first preliminary studies. However the friction factor of TBM methodology can be $40 \%$ less, and hence in energy cost, of the D\&B methodology potentially generating a trade-off worth considering.

On the other hand, having less adits is also considered an improvement since less access roads and platforms have to be developed to support their location and construction phase. Additionally building adits requires development of engineering, coordinating interference with the current mine and other administrative processes which can be very time and resource consuming. However, the number of adits is still limited by the largest available cross-section of the project defined at $9 \times 9 \mathrm{~m}$.

The last, but not least consideration of the larger study is the opportunity to have more airflow available at an earlier time. Adits take about three years to be developed considering all the processes involved until fans are running, if only one working face is considered per adit. As such, if a $9 \times 9 \mathrm{~m}$ adit is considered instead of a $7 \times 7 \mathrm{~m}$ adit, this means having $700 \mathrm{~m}^{3} / \mathrm{s}$ instead of $500 \mathrm{~m}^{3} / \mathrm{s}$ when the fan starts as presented in Table 3. However the largest economical airflow volume for the $9 \times 9 \mathrm{~m}$ cross-section is $870 \mathrm{~m}^{3} / \mathrm{s}$, according to Table 4 . The change in cross-section could deliver an excess of $200-370 \mathrm{~m}^{3} / \mathrm{s}$, which in percentage is $40-74 \%$ larger than the initial $500 \mathrm{~m}^{3} / \mathrm{s}$ considered. Taking into account the volume of metres to be developed during the 2015-2018 period which will be followed by more development faces and production, having more available airflow is an important asset for all the underground developments.

The estimated airflow requirements of the New Level Mine Project in time are presented in Table 6, until reaching full production by the year 2030. As can be observed in Table 6 , the airflow requirements steadily increase over time until reaching $4,000 \mathrm{~m}^{3} / \mathrm{s}$. This study did not consider the impact of adding these adits in time. The study was focused on determining the number of adits and cross-section required to deliver the total airflow volume of $4,000 \mathrm{~m}^{3} / \mathrm{s}$. It is expected that the final result when considering the entrance of adits displaced in time will not change the economical cross-section, which will remain valid as per the analysis presented in this study; however, this still remains to be proven. 
Another consideration to this study is the validity of the prices considered to determine the costs and the final economical cross-section. The main issue concentrates around the rise in development costs that Chilean mining companies have faced for the last four years and the increase in the energy cost and also the lack of availability. Considering that the decision has to be taken for the next 16 years, it is possible that further revision to this study will have to be performed in the future every time a new set of adits is introduced. At the same time a trade-off study will be required to determine if El Teniente mine ventilation infrastructure can be recycled to avoid building more adits as a cost effective measure.

\section{Table 6 Airflow volume requirement until reaching full production}

\begin{tabular}{cc}
\hline Year & $\mathrm{m}^{3} / \mathrm{s}$ \\
\hline 2014 & 342 \\
2015 & 950 \\
2016 & 1,254 \\
2017 & 1,496 \\
2018 & 1,397 \\
2019 & 1,735 \\
2020 & 1,934 \\
2021 & 2,175 \\
2022 & 2,599 \\
2023 & 3,068 \\
2024 & 3,342 \\
2025 & 3,613 \\
2026 & 3,712 \\
2027 & 3,851 \\
2028 & 3,893 \\
2029 & 3,927 \\
2030 & 4,000 \\
\hline
\end{tabular}

Finally, another concern is the trade-off between having eight $7 \times 7 \mathrm{~m}$ adits in the intake, with smaller cross-section area and lower risk of losing one as a result of a collapse, and having one $7 \times 7 \mathrm{~m}$ adit and five $9 \times 9 \mathrm{~m}$ adits with larger cross-section area and higher risk of losing one as a result of a collapse. The same analysis has to be developed for the exhaust. The resulting impact in terms of the remaining airflow and the time required to replace the collapsed adit is significant and should be taken into account when making the final decision.

\section{$6 \quad$ Conclusions}

This study presents a manual methodology to extend the initial economical cross-section model presented by Hartman et al. (1997) and McPherson (1993) into a manual multiple parallel economical cross-section model. The methodology was implemented with the construction and energy values obtained from the current developments of the New Level Mine Project and were applied to a total airflow volume requirement of $3,500 \mathrm{~m}^{3} / \mathrm{s}$. According to the input values used in this study, the analysis indicated that the best solution was to consider the $7 \times 7 \mathrm{~m}$ adit under construction with an airflow of $500 \mathrm{~m}^{3} / \mathrm{s}$ and five more $9 \times 9 \mathrm{~m}$ adits to be developed with an airflow of $700 \mathrm{~m}^{3} / \mathrm{s}$. As indicated in the previous section, more 
consideration is required before making the final decision, as some of the trade-off that will be generated could unbalance the economical multiple cross-sectional result. Finally, the study focused in the determination of the number of adits and their economical cross-sectional area to deliver the airflow volume of $3,500 \mathrm{~m}^{3} / \mathrm{s}$ at the intake and at the exhaust considering the problem is symmetrical. However, as the adits will be introduced in time to cover the increasing airflow requirements of the mine, validation of the proposed solution while considering actual time scales remains to be proven.

\section{References}

Acuña, EI \& Jodouin, J 2010, 'Optimization of Upper Kelly Lake Project Ventilation Design', Proceedings of the Maintenance Engineering and Mine Operators Conference: MEMO 2010, Canadian Institute of Mining, Metallurgy and Petroleum, Westmount.

Hartman, HL, Mutmansky, JM, Ramani, RV \& Wang, YJ 1997, 'Economics of Airflow', Mine Ventilation and Air Conditioning, John Wiley \& Sons, New Jersey, pp. 431-451.

McPherson, MJ 1993, Subsurface Ventilation and Environmental Engineering, Chapman \& Hall, London, pp. 263-290. 

requirements of the New Level Mine Project 